\title{
Spatial contingency and the McCollough effect
}

\author{
SHEPARD SIEGEL, LORRAINE G. ALLAN, LYNDA ROBERTS, and THOMAS EISSENBERG \\ McMaster University, Hamilton, Ontario, Canada
}

\begin{abstract}
On the basis of a conditioning analysis of the orientation-contingent color aftereffect (McCollough effect, ME), orientation stimuli become associated with simultaneously presented chromatic stimuli. This account suggests that decreasing the contingency between the grid orientation and color should decrease the strength of the aftereffect. Results of previous research indicate that decreasing the temporal contingency (by presenting homogeneous chromatic stimuli between presentations of chromatic grids) does not decrease the ME. However, it has been suggested that the appropriate contingency-degradation procedure would involve decreasing spatial (rather than temporal) contingency. That is, the illusion should be attenuated by extending the color beyond the confines of the grid. Contrary to this hypothesis, the results of the present experiments provide no evidence that decreasing the spatial contingency between grid and color decreases the $\mathrm{ME}$; rather, the aftereffect is increased by such a manipulation.
\end{abstract}

Orientation-contingent color aftereffects, originally reported by McCollough (1965), can be demonstrated readily. During an induction period of several minutes, an observer inspects two patterns that alternate every few seconds. For example, one pattern could consist of alternating black and green horizontal bars, and the other pattern could consist of alternating black and magenta vertical bars. Following such induction, complementary color aftereffects contingent on bar orientation are noted; black and white test patterns appear chromatic. In this example, the white space between black horizontal bars appears pinkish, and the white space between black vertical bars appears greenish.

A number of investigators have suggested that the McCollough effect (ME) is a type of Pavlovian conditioning (e.g., Allan \& Siegel, 1986; Bonnet, 1975; Bonnet, LeGall, \& Lorenceau, 1984; Brand, Holding, \& Jones, 1987; Murch, 1976; Schmidt, Pinette, \& Finke, 1978; Siegel \& Allan, 1985; Skowbo, Timney, Gentry, \& Morant, 1975; Sloane, Ost, Etheriedge, \& Henderlite, 1989; Westbrook \& Harrison, 1984). Generally, these investigators have adopted the conditioning analysis of the ME presented by Murch (1976):

The lined grid in inspection functions as a conditioned stimulus (CS) while color functions as the unconditioned stimulus (UCS). As a result of the pairing of the CS (lined grid) with the UCS (color) a conditioned response (CR) develops so that the adaptive response of the visual system to the color is evoked by the lined grid. (p. 615)

This conditioning interpretation of the ME is supported by demonstrations that manipulations of the putative CS

This research was supported by grants to L. G. Allan and S. Siegel from the Natural Sciences and Engineering Research Council of Canada and the United States National Institutes of Mental Health. Correspondence concerning this article should be addressed to either Shepard Siegel or Lorraine G. Allan, Department of Psychology, McMaster University, Hamilton, Ontario L8S 4K1, Canada. (grid) and UCS (hue) have conditioning-like effects; thus, the perceptual phenomenon, like Pavlovian conditioning, is subject to substantial retention, generalization, extinction, spontaneous recovery, retardation by UCS habituation, overshadowing, blocking, unblocking, and overprediction (see Allan \& Siegel, 1986; Brand et al., 1987; Sloane et al., 1989).

Skowbo and Forster (1983) evaluated another potential parallel between the ME and conditioning. They reasoned that if the ME represents an instance of Pavlovian conditioning, contingency manipulations should affect the perceptual illusion much as they affect other CRs. Generally, decreasing the contingency between a CS and a UCS decreases the strength of conditioning. Such a decrease in contingency typically is effected by UCS presentations interpolated between paired CS-UCS presentations (see Rescorla \& Wagner, 1972). On the basis of a conditioning analysis of the ME, then, it would be expected that the perceptual illusion should be degraded by presentation of the putative UCS (homogeneous chromatic stimuli) between presentations of the chromatic grids. Skowbo and Forster (1983) reported that this manipulation did not influence the magnitude of the $\mathrm{ME}$, and suggested that their results were contrary to an associative account of the phenomenon.

Skowbo and Forster's (1983) results were subsequently replicated by Siegel and Allan (1987). Siegel and Allan (1987) agreed that the ineffectiveness of interpolated chromatic stimuli to degrade the ME made associative interpretations of the phenomenon problematic. However, they also noted that this failure of contingency manipulations to affect the ME is in marked contrast to the abundant evidence indicating parallels between the ME and Pavlovian conditioning. Siegel and Allan (1987) presented an interpretation of this finding that is congenial with an associative analysis. This alternative interpretation emphasizes the fact that the ME typically is induced with simultaneous (rather than successive) presentations of grid and 
color. Indeed, sequential presentation of grid and color leads to a much smaller aftereffect than simultaneous presentation does (see, Murch, 1976).

Prevalent views of conditioning are based primarily on successive conditioning-that is, conditioning in which the onset of the CS precedes the onset of the UCS. Such views emphasize the role of the CS as a signal for the UCS-a relationship best described as "informative" or "predictive" (see Rescorla, 1981). As discussed by Rescorla and colleagues (e.g., Rescorla, 1986; Rescorla \& Durlach, 1981), "substantial, important, and perhaps qualitatively different, learning occurs when stimuli are presented simultaneously" (Rescorla, 1981, p. 78). In the case of simultaneously presented sensory events, subjects may form a unitary representation of the elements that form the compound perceptual stimulus, rather than form separate representations that are then associated (i.e., "coherence within units," rather than "coherence between units" [Asch, Ceraso, \& Heimer, 1960]).

Typically, CS-UCS contingency has been conceptualized in temporal terms; contingency is decreased by presenting the UCS at times other than when the CS is presented. This type of contingency manipulation decreases the value of the CS as a predictor of the UCS, and, as expected, it decreases the strength of conditioning involving CS-UCS pairings in a successive conditioning manner. In the case of simultaneous conditioning, however, it is likely that the subject does not learn that the CS predicts the UCS. Rather, the subject learns that the CS forms a part of the UCS. As suggested by Rescorla (1986), it is possible that temporal contingency manipulations may affect only successive associations, and not simultaneous associations. Rather, simultaneous associations can be affected by manipulation of spatial contingency.

If the ME is a manifestation of a simultaneous association, it may be decreased by decreasing the spatial contingency between grid and hue. Indeed, Rescorla (1986) reported that the strength of an association between two simultaneously presented visual stimuli in pigeons can be decreased through the manipulation of spatial contingency. Pigeons were trained to associate a color (e.g., red) with a simultaneously presented geometric form outline (e.g., a small triangle, constructed from thin black lines, presented at the center of the response key). In the high spatial contingency condition, the color was a property of the form (i.e., the interior of the triangle was red, and the rest of the response key was achromatic). In the low spatial contingency condition, the color was a property of the form and the ground on which the form occurred (i.e., the entire response key was red, and the triangle was an outline form at the center of the key). The simultaneous association was greater with the high spatial contingency.

Typically, the ME is induced with a high spatial contingency between grid and color (i.e., during induction, chromatic grids are presented on an achromatic field). In the present experiments, we evaluated the effects of decreasing the spatial contingency between grid and color on the magnitude of the ME.

\section{EXPERIMENT 1}

To reduce spatial contingency, during induction with chromatic grids, the color extended to the surrounding field, beyond the area bounded by the grids.

\section{Method}

\section{Subjects}

The subjects were 52 male and female students with no previous experience in contingent aftereffect tasks. They were not screened for color deficiencies. They were enrolled in introductory psychology at McMaster University and received course credit for their participation. The experiment was conducted in two replications. In each replication, 13 subjects were assigned to each of two groups: high spatial contingency (HSC) or low spatial contingency (LSC).

\section{Apparatus}

Temporal parameters, stimulus presentation, and recording of responses were controlled by a Tandy 3000 computer, equipped with a VGA display card. The stimuli were displayed on a Zenith "flat screen" monitor (Model 1490). The monitor was located about $125 \mathrm{~cm}$ from the subject. The color on the monitor is the combination of red $(R)$, green $(G)$, and blue $(B)$. When each input is at its maximum value $(R=G=B=63)$, the result is white. When each input is at its minimum value $(R=G=B=0)$, the result is black. Changes in the relative proportions of $R, G$, and $B$ produce various hues at different saturations.

\section{Stimuli}

To induce an orientation-contingent color aftereffect, all subjects received presentations of a horizontal grid alternating with a vertical grid. Each grid was $6.0 \times 6.4 \mathrm{~cm}$, presented at the center of the $27.5 \times 20.3 \mathrm{~cm}$ monitor screen (all dimensions are width $\times$ height). The grid subtended about $2.7^{\circ} \times 2.9^{\circ}$ of visual angle. The monitor screen subtended about $12.6^{\circ} \times 10.6^{\circ}$ of visual angle.

The grids were composed of 16 bars, either horizontal or vertical. Eight of the bars were black $(R=G=B=0)$. For the HSC condition, the horizontal black bars alternated with horizontal green bars and the vertical black bars alternated with vertical magenta bars. The remainder of the monitor screen was white. For the LSC condition, the black bars were presented on a colored field; the entire monitor screen (except for the black bars) was either green (when the grid was horizontal) or magenta (when the grid was vertical).

Chromaticity and illuminance of the induction stimuli were assessed with a Minolta Chroma Meter II Incident. Measurements were taken with the display completely illuminated with the color that was being assessed, using a procedure similar to that in Houck and Hoffman (1986). The RGB values, illuminance values (lux), and chromaticity values (CIE $x$-and $y$-coordinates) for the green and magenta grids, and the white background of the HSC condition, are shown in Table 1.

\section{Aftereffect Measurement}

The magnitude of the orientation-contingent color aftereffect was determined with a computer colorimeter, which provided a measure of the amount of color necessary to counterbalance or nullify

Table 1

RGB Values, Illuminance Values (lux), and Chromaticity Values (CIE $x$ - and $y$-coordinates) for Chromatic Grids Used During McCollough Effect Induction in Experiment 1

\begin{tabular}{lrrrccc}
\hline & $\mathrm{R}$ & $\mathrm{G}$ & $\mathrm{B}$ & Illuminance & $x$ & $y$ \\
\hline Magenta & 63 & 0 & 25 & 13.1 & .552 & .311 \\
Green & 0 & 63 & 0 & 48.9 & .309 & .601 \\
White & 63 & 63 & 63 & 62.0 & .298 & .334 \\
\hline
\end{tabular}


a color aftereffect. The procedure was a modified form of that described by Siegel and Allan (1987). The subjects were presented with a display consisting of a checkerboard design of grid patterns. Each grid was oriented differently (either horizontal or vertical) than the adjacent grid, and each of the grids was the same size and spatial frequency as the grids used during induction. This display was four grids across the screen $\times$ three grids down. The relative amounts of green and magenta color provided to the grids could be adjusted with control buttons on the computer keyboard. At the outset of a determination of the magnitude of the aftereffect, grids of one orientation were clearly pinkish, and the grids of the other orientation were clearly greenish. The subject was instructed to adjust the display such that the entire checkerboard appeared achromatic, and then press the "enter" key. A press on this key would restart the test pattern, with the alternative grids being pinkish and greenish.

To make the test pattern appear achromatic, the subject adjusts the display so that red and blue are added to the greenish grids, and green simultaneously added to the pinkish grids. In the absence of a color aftereffect, a subject should adjust the pattern to be objectively achromatic $(R=G=B=63$ ). This setting, however, would appear as a mismatch if a color aftereffect were present. Specifically, for an achromatic grid to be seen following induction with the magenta vertical and green horizontal stimuli used in this experiment, relatively less green would be added to the vertical test grids (e.g., $R=63, G=61, B=63$ ) and relatively less red and blue would be added to the test horizontal grids (e.g., $R=61, G=63$, $B=61$ ). The difference between the true achromatic setting and the subject's setting of subjective achromaticity provided an index of the color aftereffect (in this example, the aftereffect magnitude is 2).

\section{Procedure}

The subjects were tested individually in a darkened room, with the experimenter present in the room throughout the session. The subjects were instructed to look at the monitor and to keep their heads upright at all times. There were three phases: preinduction measurement of the $\mathrm{ME}, \mathrm{ME}$ induction, and postinduction measurement of the ME.

Preinduction measurement. Prior to ME induction, the subjects were presented with the checkerboard test pattern on 10 occasions. The mean of the 10 settings of the monitor color guns provided the preinduction measure. Although no time limit was imposed, the subjects generally completed this task in about $2 \mathrm{~min}$.

Induction. ME induction started immediately after the final preinduction determination. This phase of the experiment lasted $10 \mathrm{~min}$. Green horizontal stimuli and magenta vertical stimuli were alternately presented at $3-\mathrm{sec}$ intervals.

Postinduction measurement. There was a $2-\min$ period in normal room illumination between ME induction and postinduction evaluation of the aftereffect. The postinduction determination, consisting of the mean setting for 10 presentations of the test pattern, was conducted in the same manner as was the preinduction determination.

\section{Results and Discussion}

The mean preinduction and postinduction determinations of subjective achromaticity $( \pm 1 S E M)$ for the HSC and LSC groups (collapsed across replications) are presented in Figure 1. As is apparent in this figure, subjects displayed an ME. Forty-seven of the 52 subjects displayed a contingent aftereffect (i.e., greater postinduction than preinduction colorimeter settings): 25 in the LSC group, and 22 in the HSC group.

The results of the experiment were analyzed with a 2 (spatial contingency, HSC or LSC) $\times 2$ (replication, 1 or 2 ) $\times 2$ (assessment, preinduction or postinduction)

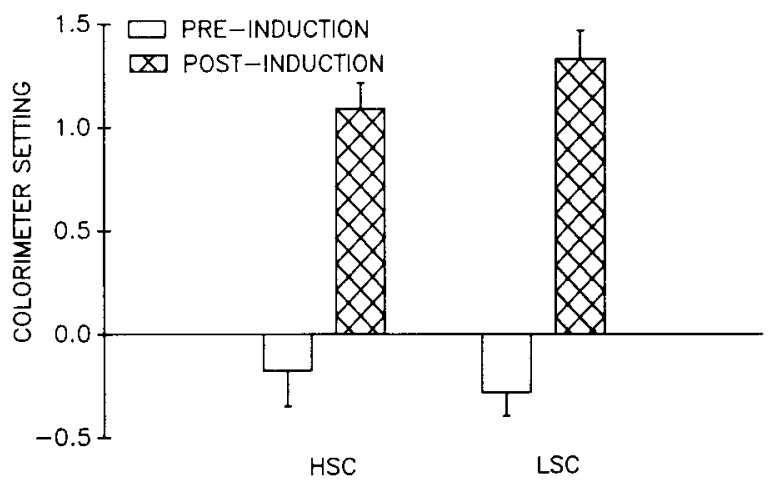

Figure 1. Mean preinduction and postinduction settings of subjective achromaticity ( \pm 1 SEM) for HSC and LSC groups (Experiment 1).

mixed-design analysis of variance (spatial contingency and replication were between-subject factors, and assessment a within-subject factor). Only the main effect of assessment was significant $[F(1,48)=98.2, p<.0001]$. That is, subjective achromaticity was different on the postinduction test from what it was on the preinduction test.

These results provide no support for the hypothesis, inspired by an associative account of the ME (see Allan \& Siegel, 1986; Siegel \& Allan, 1987), that the perceptual illusion can be decreased by decreasing the spatial contingency between grid and hue.

In this experiment, as in the experiment by Rescorla (1986), spatial contingency between pattern and color was decreased by extending the color beyond the pattern. It is possible, however, that this manipulation was not effective. Subjects may have fixated the grid in the center of the display, and not attended to the surround; they may not have "noticed" whether the surrounding area was chromatic or achromatic. The next experiment was designed to ensure that the subject was exposed to the chromatic (LSC subjects) or achromatic (HSC subjects) surround.

\section{EXPERIMENT 2}

In this experiment, spatial contingency was manipulated as in Experiment 1. However, unlike in the previous experiment, the grid was not stationary at the center of the video screen throughout ME induction; rather, its location changed in an unpredictable manner every second. Thus, in tracking the grid during ME induction, the subject was exposed to both the background and the pattern.

In Experiment 1, the ME was evaluated with the method of adjustment. In Experiment 2, the illusion was evaluated with a variant of the method of constant stimuli. We have previously reported that this procedure is a sensitive and effective measure of contingent color aftereffects in general, and of orientation-contingent color aftereffects in particular (Allan, Siegel, Collins, \& MacQueen, 1989; Allan, Siegel, Toppan, \& Lockhead, in press). 


\section{Method}

Subjects and Apparatus. The subjects were 41 students drawn from the same population as those participating in Experiment 1 .

Temporal parameters, stimulus presentation, and recording of responses were controlled by a Macintosh IIcx computer equipped with an 8-bit video display card. This system enabled 256 gradations of saturation of each hue (ranging from 0 to 255), in contrast to the 64 gradations in the previous experiment. ${ }^{2}$ The stimuli were displayed on an Apple color monitor (Model M0401PA), located about $125 \mathrm{~cm}$ from the subject.

Induction Stimuli. The stimuli consisted of either horizontal or vertical grids $\left(5.9 \mathrm{~cm}\right.$ square, subtending approximately $2.7^{\circ}$ of visual angle), presented on the $25.5 \times 19.5 \mathrm{~cm}$ video screen. The entire screen subtended approximately $11.6^{\circ} \times 8.9^{\circ}$ of visual angle. The grids were composed of 20 bars, either horizontal or vertical.

For HSC subjects, alternate bars were chromatic, with the remainder of the video screen being gray. For 10 HSC subjects, the $\mathrm{ME}$ was induced with green horizontal and magenta vertical grids (Group HSC:GH/MV). The relationship between grid orientation and hue was reversed for another 10 HSC subjects (Group HSC:GV/MH).

For LSC subjects, the entire video screen was colored. The ME was induced either with black horizontal bars on a green field and black vertical bars on a magenta field (Group LSC:GH/MV, $n=10$ ) or with the reverse relationship between grid orientation and screen color (Group LSC:GV/MH, $n=11$ ).

Induction lasted $10 \mathrm{~min}$. The subjects were presented with 120 alternating green and magenta stimuli. Each chromatic stimulus presentation lasted $5 \mathrm{sec}$, with the grid moving to a new location (randomly determined) on the video display every second.

Aftereffect measurement. Illusory color was measured both before and after ME induction (preinduction and postinduction determination, respectively). For both assessments, the subjects received 50 presentations of each grid orientation. On each presentation, the space between the black bars of the grid could be one of five colors: one of two shades of pale pink (P1 and P2, with $\mathrm{P} 2$ being more saturated than P1), one of two shades of pale green ( $G 1$ and $G 2$, with $G 2$ being more saturated than $G 1$ ), or achromatic. Grid orientation (horizontal or vertical) and color (P1, P2, $G 1, G 2$, and achromatic) were randomly ordered, with the restriction that each orientation was presented in each color 10 times. The grids were presented at the center of the video display, with the remainder of the screen being black. On each of the 100 presentations of the grid during the aftereffect determination, the subject had to make a binary response - "green" or "pink." The grid remained on the screen until the subject responded. After a response, the screen was black for $1 \mathrm{sec}$ before the next test grid was presented.

Prior to the start of the experiment, the subjects were familiarized with the color judgment task. Before preinduction aftereffect determination, the subjects received 16 practice trials. For each practice trial, the grid was one of the four unsaturated hues $(G 1$, $G 2, P 1$, or $P 2$ ) and either horizontal or vertical. Each of the eight hue-orientation combinations was presented twice (in random order). In contrast to the preinduction and postinduction assessments, feedback was provided on these practice trials. Immediately after making a "green" or "pink" response, the subjects were informed that their response was "correct" or "error" via the speech-synthesis capability of the computer. These practice data were ignored.

Chromaticity and illuminance were assessed in the manner described previously. The RGB values, illuminance values (lux), and chromaticity values (CIE $x$ - and $y$-coordinates) for the green and magenta grids, the gray background of the HSC condition, and the various hues used to measure the ME, are shown in Table 2.
Table 2

RGB Values, Illuminance Values (lux), and Chromaticity Values (CIE $x$ - and $y$-coordinates) for Stimuli Used in Experiment 2

\begin{tabular}{lrrrrrr}
\hline & R & G & B & Illuminance & $x$ & $y$ \\
\hline Induction Stimuli & & & & & & \\
$\quad$ Magenta & 255 & 0 & 255 & 16.4 & .274 & .132 \\
Green & 0 & 255 & 0 & 43.3 & .271 & .609 \\
Gray background & 252 & 252 & 252 & 57.3 & .272 & .302 \\
Assessment Stimuli & & & & & & \\
P2 & 255 & 238 & 238 & 54.5 & .279 & .302 \\
P1 & 255 & 245 & 245 & 57.4 & .277 & .301 \\
Achromatic & 252 & 252 & 252 & 57.3 & .272 & .302 \\
G1 & 245 & 255 & 245 & 58.4 & .272 & .308 \\
G2 & 238 & 255 & 238 & 56.4 & .271 & .314 \\
\hline
\end{tabular}

\section{Results and Discussion}

The psychometric function relating the probability of the subject's reporting that the grid appeared green, $P(\mathrm{G})$, to the physical characteristics of the assessment stimulus (ranging from P2 to G2) was determined. All 41 subjects displayed shifts from preinduction to the postinduction psychometric functions indicative of a ME.

Figure 2 displays the mean preinduction and postinduction functions for HSC and LSC groups that received $\mathrm{ME}$ induction with green and magenta stimuli. For simplicity in presentation, the psychometric functions displayed in Figure 2 are collapsed across the dimension of grid orientation (e.g., the functions labeled HSC-green present the combined assessment data for the horizontal grids for Groups HSC:GH/MV and the vertical grids for Group HSC:GV/MH).

The preinduction functions were similar for all groups. The functions moved apart after ME induction, with the function for the magenta stimuli being higher than the function for the green stimuli. Moreover, for both hues, there was a tendency for greater MEs in LSC groups than in HSC groups.

For purposes of statistical analysis, the difference between the number of "green" responses to the orientation paired with green and to the orientation paired with magenta was determined. The mean difference scores over the five test hues were subjected to a mixed-design analysis of variance. The between-subject factors were spatial contingency (HSC or LSC) and induction-color-orientation pairing (GH/MV or $\mathrm{MV} / \mathrm{GH})$. The within-subject factor was assessment (preinduction or postinduction). The only significant effects were assessment $[F(1,37)=844, p<$ $.0001]$, and the interaction between this variable and spatial contingency $[F(1,37)=7.83, p<.01]$. That is, an $\mathrm{ME}$ was induced, and the magnitude of the ME was larger for subjects in the LSC group than for subjects in the HSC group.

In summary, in Experiment 2, as in Experiment 1, there was no evidence that decreasing the spatial contingency between grid and hue decreased the ME. Indeed, in Experiment 2, in which an attempt was made to expose subjects to both the chromatic grid and the chromatic 

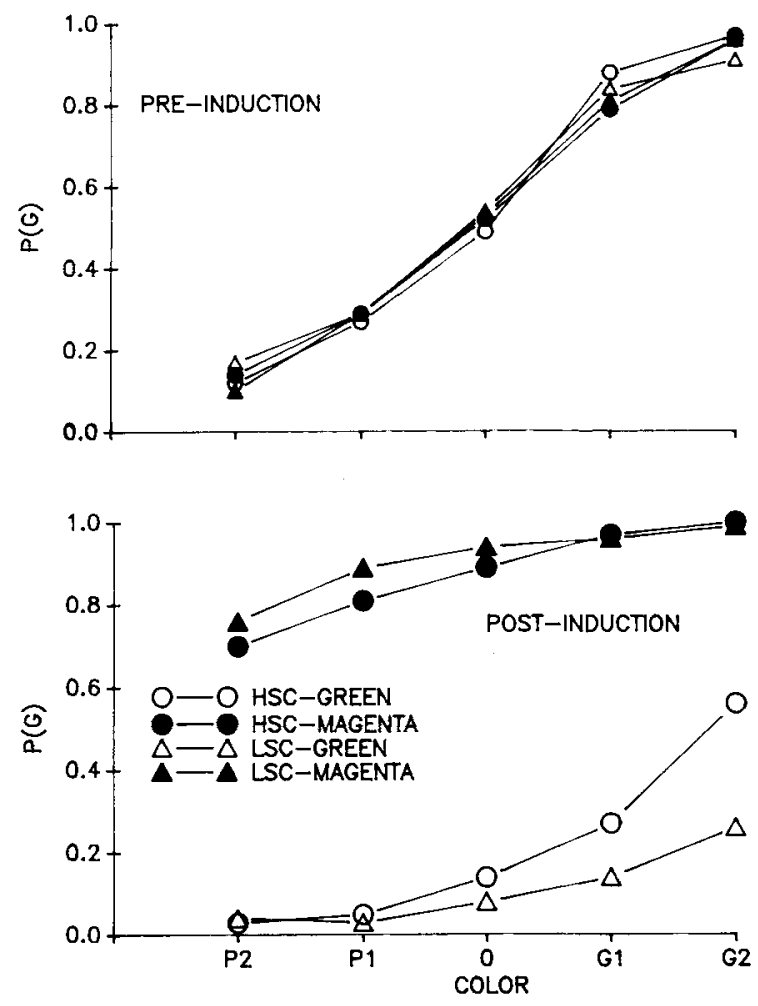

Figure 2. Mean preinduction and postinduction psychometric functions for HSC and LSC groups that received ME induction with green and magenta stimuli (Experiment 2). The probability that the subject reported the test stimulus as green, $P(G)$, is plotted against the color of the test stimulus: P2 and P1 are two shades of pink, with $P 2$ being more saturated than P1; G2 and G1 are two shades of pale green, with $\mathbf{G} 2$ being more saturated than $\mathbf{G 1 ;} 0$ is achromatic.

(Group LSC) and achromatic (Group HSC) background, there was a significantly greater contingent aftereffect in the LSC group.

\section{GENERAL DISCUSSION}

It has previously been reported that the magnitude of the ME is not reduced by decreasing the temporal contingency between grid and color (Siegel \& Allan, 1987; Skowbo \& Forster, 1983), suggesting that the perceptual phenomenon is not an instance of Pavlovian conditioning. However, Siegel and Allan (1987) noted that the ME typically is induced by simultaneous presentations of grid and hue. They hypothesized that temporal contingency manipulations may not be relevant to evaluating associations resulting from simultaneous CS and UCS presentations. Rather, such simultaneous associations should be affected by spatial contingency manipulations (Rescorla, 1986). The results of these experiments provide no support for this hypothesis.

In the present experiments, as in the experiments of Rescorla (1986), spatial contingency between pattern and color was decreased by extending the color beyond the pattern. In Experiment 1, the aftereffect was measured by the method of adjustment. The group with the low spatial contingency between the grid and color (i.e., the color extended beyond the grid pattern, presented at the center of the video display, to the entire video display) demonstrated an insignificantly greater aftereffect than did the group with the high spatial contingency between grid and color (i.e., the color was confined to the grid pattern, with the rest of the video display being gray).

In Experiment 2, the aftereffect was measured with the method of constant stimuli. In addition, an attempt was made to ensure that subjects noticed the video display beyond the grid during ME induction by moving the chromatic grid around the chromatic (LSC) or achromatic (HSC) surround. Once again, there was no evidence that decreasing the spatial contingency between grid and color decreased the ME. In fact, the LSC group displayed a significantly greater ME than did the HSC group. These results provide no support for an associative account of the ME.

The fact that the ME is greater following LSC than HSC induction suggests that the magnitude of the illusion is related to the quantity of color present at the time of grid presentation. Thus, the present findings, indicating that the ME is enhanced by increasing the area of color presented at the time of grid presentation, is similar to earlier findings demonstrating that the $\mathrm{ME}$ is enhanced by increasing the saturation of the color (e.g., White, 1978).

\section{REFERENCES}

AllaN, L. G., \& SIEGEL, S. (1986). McCollough effects as conditioned responses: Reply to Skowbo. Psychological Bulletin, 100, 388-393.

Allan, L. G., Siggel, S., Collins, J. C., \& MacQueen, G. M. (1989). Color aftereffect contingent on text. Perception \& Psychophysics, 46, 105-113.

allan, L. G., Siegel, S., Toppan, P., \& Lockhead, G. (in press). Assessment of the McCollough effect by a shift in psychometric function. Bulletin of the Psychonomic Society.

Asch, S. E., Ceraso, J., Heimer, W. (1960). Perceptual conditions of association. Psychological Monographs, 74 (No. 490), 1-48.

BoNNET, C. (1975). Le conditionnement sensori-sensoriel dans les analyseurs visuels d'attributs. Psychologie Française, 20, 34-43.

Bonnet, C., LeGall, M., LoRenceaU, J. (1984). Visual motion aftereffects: Adaptation and conditioned processes. In L. Spillmann \& B. R. Wooten (Eds.), Sensory experience, adaptation, and perception (pp. 561-582). Hillsdale, NJ: Erlbaum.

Brand, J. L., Holding, D. H., \& Jones, P. D. (1987). Conditioning and blocking of the McCollough effect. Perception \& Psychophysics, 41, 313-317.

HoucK, M. R., Hoffman, J. E. (1986). Conjunction of color and form without attention: Evidence from an orientation-contingent color aftereffect. Journal of Experimental Psychology: Human Perception \& Performance, 12, 186-199.

McCollough, C. (1965). Color adaptation of edge detectors in the human visual system. Science, 149, 1115-1116.

MurCH, G. M. (1976). Classical conditioning of the McCollough effect: Temporal parameters. Vision Research, 16, 615-619.

Rescorla, R. A. (1981). Simultaneous associations. In P. Harzem \& M. D. Zeiler (Eds.), Predictability, correlation, and contiguity (pp. 4780). New York: Wiley.

Rescorla, R. A. (1986). Two perceptual variables in within-event learning. Animal Learning \& Behavior, 14, 387-392. 
Rescorla, R. A., \& Durlach, P. J. (1981). Within-event learning in Pavlovian conditioning. In N. E. Spear \& R. R. Miller (Eds.), Information processing in animals: Memory mechanisms (pp. 81-111). Hillsdale, NJ: Erlbaum.

Rescorla, R. A., W Wagner, A. R. (1972). A theory of Pavlovian conditioning: Variations in the effectiveness of reinforcement and nonreinforcement. In A. H. Black \& W. F. Prokasy (Eds.), Classical conditioning II: Current theory and research (pp. 64-99). New York: Appleton-Century-Crofts.

SChmidt, M. J., Pinette, P. R., \& Finke, R. A. (1978). Further evidence for conditioning processes in the McCollough effect. Journal of General Psychology, 99, 117-132.

Siegel, S., AllaN, L. G. (1985). Overshadowing and blocking of the orientation-contingent color aftereffects: Evidence for a conditioning mechanism. Learning \& Motivation, 16, 125-138.

Siegel, S., AllaN, L. G. (1987). Contingency and the McCollough effect. Perception \& Psychophysics, 42, 281-285.

Skowbo, D., \& Forster, T. (1983). Further evidence against the classical conditioning model of McCollough effects. Perception \& Psychophysics, 34, 552-554.

Skowbo, D., Timney, B. N., Gentry, T. A., \& Morant, R. B. (1975). McCollough effects: Experimental findings and theoretical accounts. Psychological Bulletin, 82, 497-510.
Sloane, M. E., Ost, J. W., Etheriedge, D. B., \& Henderlite, S. E. (1989). Overprediction and blocking in the McCollough effect. Perception \& Psychophysics, 45, 110-120.

WESTBROOK, R. F., \& HARRISON, W. (1984). Associative blocking of the McCollough effect. Quarterly Journal of Experimental Psychology, 36A, 309-318.

WHITE, K. D. (1978). Studies of form-contingent color aftereffects. In J. C. Armington, J. Krauskopf, \& B. R. Wooten (Eds.), Visual psychophysics and physiology (pp. 267-289). New York: Academic Press.

\section{NOTE}

1. With the Macintosh IIcx computer, colors are selected with the "Color Picker Dialog Box" in the Color Control Panel. This utility displays levels of saturation of each hue as a number ranging from 0 (black) to 65,535 (full saturation). With an 8-bit video display card, this means that each of the 256 levels of saturation is displayed in step sizes of 256. Thus, the relationship between the values indicated in the Macintosh IIcx "Color Picker" (C) and the saturation levels indicated here $(S)$ is expressed by the formula: $\mathrm{C}=256 \mathrm{~S}+255$.

(Manuscript received February 27, 1990; revision accepted for publication May 15, 1990.) 